This is a post-peer-review, pre-copyedit version of an article published in IEEE transactions on antennas and propagation. The final authenticated version is available online at:

DOI http://dx.doi.org/10.1109/TAP.2018.2800802

(C) 2018 IEEE. Personal use of this material is permitted. Permission from IEEE must be obtained for all other uses, in any current or future media, including reprinting/republishing this material for advertising or promotional purposes, creating new collective works, for resale or redistribution to servers or lists, or reuse of any copyrighted component of this work in other works. 


\title{
Upper Bounds on the Bandwidth of Electrically Small Single Resonant UHF-RFID Tags
}

\author{
Gerard Zamora, Member, IEEE, Simone Zuffanelli, Member, IEEE, Pau Aguilà, Student Member, IEEE, \\ Ferran Paredes, Member, IEEE, Ferran Martín, Fellow, IEEE, and Jordi Bonache, Member, IEEE
}

\begin{abstract}
In this paper, the upper limits on the bandwidth of single resonant UHF-RFID tags as a function of the tag size are investigated, with and without forcing perfect matching between the antenna and the application specific integrated circuit (ASIC). By means of a circuit network analysis, it is found that bandwidth upper bounds of small tags are significantly higher in comparison with considering conjugate matching. Particularly, it is shown that the half-power bandwidth is $\sqrt{2}$ times (approximately $41 \%$ ) higher, requiring a proper relaxation of the matching level at resonance. It is also shown that bandwidth of small real tags with perfect matching, which is typically far from its upper bound, can also be enhanced approximately the same factor at the expense of a small reduction $(13.4 \%)$ in the peak read range. A practical example is provided where two small SRR-based tags of same size $\left(k_{0} a=0.31\right)$ are designed. It demonstrates that such improvement on the tag bandwidth can be approximately obtained by simply changing the chip position, without the need of an external matching network. The improved tag was fabricated and measured, as a proof of concept. The results obtained from the proposed analysis allow RFID designers to determine how well a tag performs, compared to theoretical bandwidth limits.
\end{abstract}

Index Terms - Antennas, bandwidth, quality factor, radio frequency identification (RFID), RFID tags, split-ring resonator (SRR).

\section{INTRODUCTION}

Radio frequency identification (RFID) is a rapidly developing technology that provides objects tagging and tracking capability by means of electromagnetic waves [1]. Typical applications of this technology are asset identification, retail item management, access control, animal tracking and vehicle security, among others. Passive tags operating at the UHF-RFID frequency band are especially employed for this kind of applications. A passive tag consists of an antenna matched to an application specific integrated circuit (ASIC), which contains the information about the tagged item. According to the current regulations for passive UHF-RFID systems [2], the frequency range comprised between $864 \mathrm{MHz}$ and $928 \mathrm{MHz}$ is operated within all the countries allocations authorized for RFID applications. In the last years, the cost of tags has experienced a significant decrease, mostly due to the development in semiconductor technology and mass production. This has involved an increase of the number of applications where RFID becomes a profitable solution.

One of the most challenging aspects regarding passive UHF-RFID tag design is size reduction. Since RFID chips are always much smaller than tag antennas, tag dimensions are determined by the antenna size, or the antenna plus the matching network when it is present. The most commonly used technique for tag size reduction consists in antenna meandering [3], [4]. However, as it is well known, size reduction leads to a trade-off between efficiency and bandwidth, especially when electrically small antennas (ESAs) are

This work was supported by MINECO-Spain (TEC2013-40600-R, TEC2016-75650-R), Generalitat de Catalunya (2014SGR-157), Institució Catalana de Recerca i Estudis Avançats (awarded F. Martín), and by ERDF.

The authors are with GEMMA/CIMITEC (Departament d'Enginyeria Electrònica), Universitat Autònoma de Barcelona. 08193 BELLATERRA (Barcelona). Spain. E-mail: Gerard.Zamora@uab.cat. treated [5], [6]. An antenna is considered to be small when $k_{0} a<0.5$ according to [5], [6], where $k_{0}$ denotes the resonance wavenumber, and $a$ is the radius of an imaginary sphere circumscribing the largest dimension of the antenna (henceforth called antenna size for the sake of brevity). Bandwidth limitations of resonant antennas are closely related to the antenna quality factor $(Q)$, which is required to be minimized for bandwidth enhancement [6]-[9]. The $Q$ of a tuned (to have zero reactance at the resonance frequency $\omega_{0}$ ) antenna is defined as the quotient between the power stored in the reacted field and the accepted power (power loss within the antenna plus radiated power). This definition presumes that the antenna may be tuned by an external matching circuit to provide a real input impedance at the operation frequency [6], [8]. Yaghjian and Best [8] derived the relationship between bandwidth and $Q$ of single resonant antennas under conjugate matching condition. However, unlike conventional antennas, RFID antennas must be matched to an ASIC having a complex input impedance, rather than a purely resistive port. This produces a direct effect upon tag bandwidth upper bounds.

In [10], the equivalent circuit network required to achieve the upper limit of the area under the return loss curve (determined by the Bode criterion [11]) was obtained for single resonant tags with perfect matching between the chip and the antenna. Meaning that maximum bandwidth at all matching levels was obtained under conjugate matching condition. The present paper shows, by means of a circuit network analysis, that such bandwidth can only theoretically be achieved by using antennas that are not electrically small. Moreover, upper bounds on the bandwidth of single resonant UHFRFID tags, with and without forcing perfect matching, are inferred as a function of the tag size. It is also shown that the bandwidth of small real tags with conjugate matching can be considerably enhanced by means of a proper reduction of the matching level at resonance, without necessarily involving an increase in the tag size.

\section{UPPER BOUNDS ON THE TAG BANDWIDTH}

The input impedance of an UHF-RFID ASIC can be modeled by a parallel combination of a conductance $G_{c}$ and a capacitance $C_{c}$ [12]. Let us consider an inductive antenna (or antenna plus matching network) with an equivalent circuit model consisting of a parallel combination of a general antenna susceptance $B_{a}$ and a frequency dependent conductance $G_{a}$, tuned at a frequency $\omega_{0}$ by the chip capacitance. The antenna conductance $G_{a}=G_{r}+G_{l}$ is modeled as a parallel combination of the radiation antenna conductance $G_{r}$ and the loss conductance $G_{l}$ (that accounts for the conductive and dielectric losses of the antenna). To maintain generality, both $G_{r}$ and $G_{l}$ are assumed to be frequency dependent. The efficiency of the antenna is related to the elements of the antenna equivalent circuit model by $\eta=G_{r} / G_{a}$ [13]. The quality factor of a general one port lossy linear antenna tuned at $\omega_{0}$ (presenting a single sufficiently isolated resonance within its operating bandwidth) with $Q \gg>1$ ( $Q$ greater than 2 usually suffices) can be accurately approximated from the antenna input impedance by [8] 


$$
Q \approx Q_{Z}=\frac{\omega_{0}}{2 R_{0}\left(\omega_{0}\right)}\left|\mathrm{Z}_{0}^{\prime}\left(\omega_{0}\right)\right|
$$

where $Z_{0}{ }^{\prime}\left(\omega_{0}\right)=R_{0}{ }^{\prime}\left(\omega_{0}\right)+j X_{0}{ }^{\prime}\left(\omega_{0}\right)$ is the frequency derivative of the input impedance of the tuned antenna (assuming $C_{c}$ to be the tuning element) at $\omega_{0}, R_{0}{ }^{\prime}\left(\omega_{0}\right)$ and $X_{0}{ }^{\prime}\left(\omega_{0}\right)$ being the frequency derivative of the input resistance $R_{0}$ and reactance $X_{0}$ of the tuned antenna at $\omega_{0}$, respectively. It is clear from (1) that minimum $Q$ (required for bandwidth broadening) is obtained by reducing $Z_{0}{ }^{\prime}\left(\omega_{0}\right)$, which can be written in terms of the conductance $G_{0}$ and susceptance $B_{0}$ of the tuned antenna using $R_{0}{ }^{\prime}\left(\omega_{0}\right)=-G_{0}{ }^{\prime}\left(\omega_{0}\right) / G_{0}{ }^{2}\left(\omega_{0}\right)$, $X_{0}{ }^{\prime}\left(\omega_{0}\right)=-B_{0}{ }^{\prime}\left(\omega_{0}\right) / G_{0}{ }^{2}\left(\omega_{0}\right)$. Because the tuning capacitor is connected in parallel with the antenna, $G_{0}=G_{a}$ and $B_{0}=B_{a}+B_{c}\left(B_{c}=\omega_{0} C_{c}\right.$ being the chip susceptance at $\omega_{0}$ ). Thus, assuming a frequency independent antenna conductance makes $R_{0}{ }^{\prime}\left(\omega_{0}\right)=0$. Whereas, $X_{0}{ }^{\prime}\left(\omega_{0}\right)$ is minimized making $B_{a}^{\prime}\left(\omega_{0}\right)$ as small as possible. As it was pointed out in [10], the Foster's reactance theorem [14] holds for the antenna equivalent circuit network required to obtain a single tag resonance with proper impedance matching between the chip and the antenna, i.e., $B_{a}{ }^{\prime}\left(\omega_{0}\right)>0$. The Foster network that provides a certain negative susceptance (inductance behavior) at a given frequency with the lowest frequency derivative of such susceptance is simply an inductor $\left(L_{a}\right)$ [15]. This leads to $B_{0}{ }^{\prime}\left(\omega_{0}\right)=2 C_{c}$ and $Q_{\mathrm{Z}}=\omega_{0} C_{c} / G_{a}$.

There is a well-known lower bound on the $Q$ for a general singlemode (fundamental TE or TM mode) tuned antenna, the Chu limit, which is related to the value of $k_{0} a$, namely $Q_{\mathrm{Chu}}=\eta\left[\left(k_{0} a\right)^{-3}+\left(k_{0} a\right)\right]$ [16]. Chu considered electrically small antennas, but later Sievenpiper et al. [17] showed that it serves as a good design guideline even for non-ESAs. To achieve a $Q$ close to that limit, the antenna must fully occupy the spherical volume defined by $k_{0} a$ [18]. However, most UHF-RFID antennas must fit in a planar shape and, therefore, the antenna $Q$ cannot approach to the Chu bound as closely as in the case of considering antennas with a spherical shape. Recently, Gustafsson et al. [19], [20] derived a new limit for antennas of arbitrary shape using a "sum rule". In [21], Yaghjian and Stuart used a quasi-static analysis to obtain a bound on $Q$ in terms of antenna volume and static polarizability. Lately, Mohammandpour Aghdam et al. [22] applied the result given in [21] to planar structures (i.e., the volume tends to zero) with rectangular shape. From [22], the theoretical lower bound on $Q$ that can be achieved by a linearly polarized planar tuned antenna can be written as

$$
Q_{\mathrm{lb}}=\eta \frac{\zeta}{\left(k_{0} a\right)^{3}},
$$

where $\zeta$, can be considered a structural penalty which depends on the antenna geometry. Gustafsson et al. found in [20] that the minimum value for $\zeta(9 \pi / 8)$ can only be obtained by means of planar antennas presenting a circular disk shape with radius $a$. However, in real life, most UHF-RFID tag antennas are enclosed by a rectangular shape and provide a single pure linear polarization (typically a single TM mode) in the direction along the length. The minimum structural penalty for this kind of antennas is around $\zeta=5.2$ (considering directivity 1.5, according to Gustafsson conditions [20]), which occurs for an optimum aspect ratio (i.e., length to width ratio) of 1.84 [22]. Notice that for a fixed efficiency, $Q_{\mathrm{lb}}$ increase rapidly as $k_{0} a$ decreases. In order to satisfy $Q \geq Q_{\mathrm{lb}}, B_{0}{ }^{\prime}\left(\omega_{0}\right)$ must be increased (with respect to $2 C_{c}$ ) for very low $k_{0} a$, by adding an additional shunt element in the tag circuit model. The simplest network able to raise $B_{0}{ }^{\prime}\left(\omega_{0}\right)$ maintaining the tag resonance at $\omega_{0}$ is a single capacitor, named here $C_{a}$. Thus, an $R L C$ parallel-circuit antenna model, cascaded to the ASIC, is to be considered to obtain the upper bounds on the bandwidth of RFID tags (see Fig. 1).

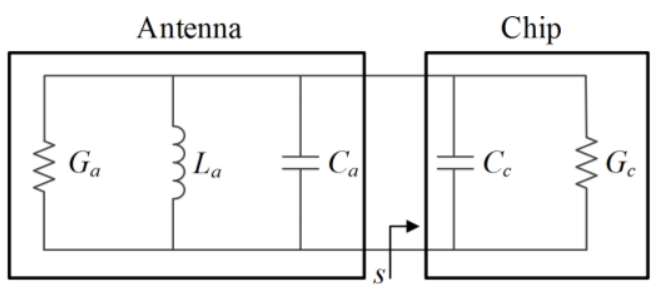

Fig. 1. Equivalent circuit model of single resonant UHF-RFID tags based on an $R L C$ parallel-circuit antenna model.

\section{A. Upper Bounds under Conjugate Matching Condition}

By isolating $B_{0}$ from the power reflection coefficient $|s|^{2}$ between the chip and the antenna in Fig. 1 [23]

$$
|s|^{2}=\frac{\left(G_{c}-G_{a}\right)^{2}+B_{0}^{2}}{\left(G_{c}+G_{a}\right)^{2}+B_{0}^{2}},
$$

it is found that there are two solutions for a certain value of the power reflection coefficient $|s|^{2}=\alpha$ :

$$
B_{0}= \pm \sqrt{2 \gamma G_{a} G_{c}-G_{a}^{2}-G_{c}^{2}}, \quad \gamma=\frac{1+\alpha}{1-\alpha} \geq 1
$$

Forcing the left hand side of (4) to be the susceptance of a parallel combination of a capacitance $C_{p}=C_{a}+C_{c}$ (i.e., parallel connection of $C_{c}$ and $C_{a}$, according to Fig. 1) and an inductance $L_{a}=1 /\left(\omega_{0}^{2} C_{p}\right)$ (to achieve tag resonance at $f_{0}$ ), the resulting fractional bandwidth evaluated at $\alpha$ can be written as

$$
\mathrm{FBW}=\frac{1}{Q} \sqrt{2 \gamma \frac{G_{c}}{G_{a}}-\left(\frac{G_{c}}{G_{a}}\right)^{2}-1},
$$

where $Q=\omega_{0} C_{p} / G_{a}$, see for example [24]. By forcing $G_{a}=G_{c}$ in (5), it is clear that upper bounds under perfect matching condition are achieved if $Q=Q_{\mathrm{lb}}$. Such a condition requires the antenna capacitance to be $C_{a}=\left(G_{c} Q_{\mathrm{lb}} / \omega_{0}\right)-C_{c}$. Thus, upper bound on bandwidth increases with the antenna size (as expected from the literature) while $C_{a}$ decreases. However, it achieves its maximum value when $Q$ reaches $Q_{c}$ (the chip quality factor evaluated at $\omega_{0}$ given by $Q_{c}=\omega_{0} C_{c} / G_{c}$ [24]) and consequently $C_{a}$ vanishes. The resulting circuit model corresponds to the ideal circuit for bandwidth broadening obtained in [10]. This leads to

$$
\mathrm{FBW}_{\text {conj,ub }}= \begin{cases}\frac{\sqrt{2 \gamma-2}}{Q_{\mathrm{lb}}} & Q_{\mathrm{lb}} \geq Q_{c} \\ \frac{\sqrt{2 \gamma-2}}{Q_{c}} & Q_{\mathrm{lb}} \leq Q_{c}\end{cases}
$$

It is worth mentioning that (6a) perfectly agrees with the relationship between bandwidth and $Q$ derived in [8], and (6b) is in agreement with the $-3 \mathrm{~dB}$ maximum bandwidth inferred in [10]. Notice that $\left(k_{0} a\right)_{\text {conj }}=\left(\eta \zeta / Q_{c}\right)^{1 / 3}$ (inferred by forcing $Q_{\mathrm{lb}}=Q_{c}$ ) corresponds to the minimum antenna size required to achieve maximum bandwidth under perfect matching condition, given by (6b). Such bandwidth can only be reached by non-ESAs, since $\left(k_{0} a\right)_{\text {conj }}>0.5$ for typical tags $\left(\zeta=5.2, Q_{c} \sim 10\right)$ with $\eta>0.1$. For lower sizes, bandwidth upper bounds are determined by the antenna, whereas for higher sizes by the ASIC.

\section{B. Upper Bounds Compared to the Conjugate Matching Case}

Maximization of tag bandwidth can be thought as a constrained optimization problem in which the fractional bandwidth (5) becomes a function of two variables $\left(G_{\mathrm{a}}\right.$ and $C_{\mathrm{a}}$ ), subject to the following three constraints: $Q \geq Q_{\mathrm{lb}}, G_{a}>0$ and $C_{a} \geq 0$. Such inequalities are referred to as the Karush-Kuhn-Tucker (KKT) conditions for the stated 
TABLE I

ELEMENTS OF THE IDEAL $R L C$ Parallel-Circuit ANTENNA Model

\begin{tabular}{ccccc}
\hline \hline Region & $\boldsymbol{G}_{\boldsymbol{a}}$ & $\boldsymbol{C}_{\boldsymbol{a}}$ & $\boldsymbol{L}_{\boldsymbol{a}}$ & $\boldsymbol{Q}$ \\
\hline I (ESA) & $\frac{G_{c}}{\gamma}$ & $\frac{G_{c} Q_{\mathrm{lb}}}{\gamma \omega_{0}}-C_{c}$ & $\frac{1}{\omega_{0}^{2}\left(C_{c}+C_{a}\right)}$ & $Q_{\mathrm{b}}$ \\
II & $\frac{\omega_{0} C_{c}}{Q_{\mathrm{bb}}}$ & 0 & $\frac{1}{\omega_{0}^{2} C_{c}}$ & $Q_{\mathrm{b}}$ \\
III & $\gamma G_{c}$ & 0 & $\frac{1}{\omega_{0}^{2} C_{c}}$ & $\frac{Q_{c}}{\gamma}$ \\
\hline \hline
\end{tabular}

generalization of the method of the Lagrange multipliers), three different set of solutions for the variables $G_{a}$ and $C_{a}$ were found, as a function of $Q_{\mathrm{lb}}$ or, equally, $k_{0} a$ (see Appendix). The upper bounds for the tag fractional bandwidth were found to be

$$
\mathrm{FBW}_{\mathrm{ub}}=\left\{\begin{array}{lc}
\frac{\sqrt{\gamma^{2}-1}}{Q_{\mathrm{b}}} & Q_{\mathrm{lb}} \geq \gamma Q_{c} \\
\sqrt{\frac{2 \gamma}{Q_{\mathrm{lb}} Q_{c}}-\frac{1}{Q_{\mathrm{lb}}^{2}}-\frac{1}{Q_{c}^{2}}} & \frac{Q_{c}}{\gamma} \leq Q_{\mathrm{lb}} \leq \gamma Q_{c} \\
\frac{\sqrt{\gamma^{2}-1}}{Q_{c}} & Q_{\mathrm{lb}} \leq \frac{Q_{c}}{\gamma}
\end{array}\right.
$$

The constituent elements of the $R L C$ parallel-circuit antenna model, as well as the $Q$ of the tuned antenna, required to achieve the upper bounds on the tag bandwidth are summarized in Table $\mathrm{I}^{1}$. It can be demonstrated that the three different regions in (7), as well as the corresponding boundaries, collapse to (6) when $\gamma$ tends to 1 (or, equivalently, $\alpha$ tends to 0 ). For $\gamma>1$ (i.e., bandwidth upper bounds are considered at $\alpha>0),\left(k_{0} a\right)_{\text {conj }}$ splits into $\left(k_{0} a\right)_{1}=\left(\eta \zeta / \gamma Q_{c}\right)^{1 / 3}$ and $\left(k_{0} a\right)_{2}=\left(\eta \zeta \gamma / Q_{c}\right)^{1 / 3}$, and consequently an intermediate region (called here region II) appears. As $\gamma$ increases, this region becomes wider, and bandwidth upper bounds separates more and more towards higher values with respect to the conjugate matching case, except at $\left(k_{0} a\right)_{\text {conj. }}$. A plot representation of (6) and (7) for $\alpha=1 / 2$ (half-power bandwidth) and $\zeta=5.2$, using the Alien Higgs 3 (SOT-323 packaging) commercial chip, is showed in Fig. 2(a) for further comprehension. It was considered $\eta=0.3$ in order to show the three different regions in a $0<k_{0} a<1$ plot. It can be seen that both bandwidths are equal at $\left(k_{0} a\right)_{\text {conj, }}$, as deduced by comparing (6) and (7). Thus, it corresponds to the unique antenna size that requires perfect matching at resonance to achieve bandwidth upper bounds at all matching levels. Within region I, $Q=Q_{\mathrm{lb}}$ is achieved by gradually reducing the antenna capacitance $C_{a}$ as $k_{0} a$ increases, until it is canceled at $\left(k_{0} a\right)_{1}$. Whereas, in region II the same condition for $Q$ is obtained by increasing the antenna conductance with $k_{0} a$ from $G_{c} / \gamma$ to $\gamma G_{c}$, going through $G_{c}$ at $\left(k_{0} a\right)_{\text {conj. }}$ Therefore, besides $\left(k_{0} a\right)_{\text {conj }}$, bandwidth upper bounds at a certain matching level requires sacrificing perfect matching at resonance.

Lowering the efficiency of the antenna involves a displacement of the boundaries $\left(k_{0} a\right)_{1},\left(k_{0} a\right)_{2}$, and $\left(k_{0} a\right)_{\text {conj }}$ towards lower values of $k_{0} a$, as well as an increase of (6a), (7a) and (7b), while the maximum upper bounds (6b) and (7c) remain constant. Thus, bandwidth upper bounds of small tags (i.e., tags based on ESAs) increase by decreasing $\eta$, as expected from the well-known trade-off between efficiency and bandwidth [17]. However, small tags with relatively high values of the antenna efficiency are of special interest in RFID, since they provide higher read ranges and size reduction. Assuming small tags based on efficient antennas and typical RFID chips $\left(Q_{c} \sim 10\right)$, we find that bandwidth upper bounds evaluated at $\alpha \leq 1 / 2$

${ }^{1}$ It is worth mentioning that the antenna conductance in the ESA region agrees with equation (16) in [27], where the circuit of Fig. 1 was considered for bandwidth maximization of single-tuned T-match-dipole tags.
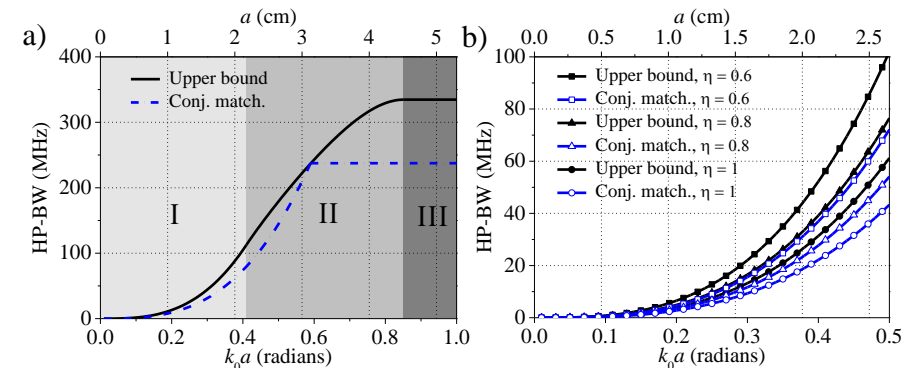

Fig. 2. Upper bounds on the tag half-power bandwidth against antenna size with (blue) and without (black) forcing conjugate matching, considering the Alien Higgs 3 and $\zeta=5.2$ in the (a) three regions (light gray, gray and dark gray, respectively) with $\eta=0.3$, and (b) ESA region for $\eta=0.6,0.8$ and 1 .

are given by (6a) and (7a). This result comes from the fact that region I extends beyond the limit for ESAs, i.e., $\left(k_{0} a\right)_{1}>0.5$. Thus, it seems reasonable to identify (7a) as the ESA region. A comparison between (6) and (7) within the ESA region

$$
\frac{\mathrm{FBW}_{\mathrm{ub}}}{\mathrm{FBW}_{\mathrm{conj,ub}}}=\sqrt{\frac{\gamma+1}{2}}
$$

reveals that bandwidth upper bounds of small tags are significantly higher (for a relatively large value of $\gamma$ ) with respect to the case of considering conjugate matching. However, as mentioned previously, it requires a reduction of the matching level at resonance, namely $|s|^{2}\left(\omega_{0}\right)=\alpha_{0}^{2} \quad$ (inferred by evaluating (3) at $f_{0}$ where $B_{0}=0$ ). Specifically, the half-power bandwidth results in a factor of $\sqrt{2}$ (approximately $41 \%$ ) higher, at the expense of reducing the matching level at resonance to 1/4. Fig. 2(b) shows a plot representation of the upper bounds on the half-power bandwidth of small tags based on efficient ESAs and the Alien Higgs 3 chip. Notice that bandwidth upper bounds are governed by (6a) and (7a), when high efficiencies are considered, and increase by lowering $\eta$.

From the previous analysis, we conclude that the antenna determines bandwidth upper bounds of small tags, whereas both the ASIC and the antenna determine bandwidth upper bounds for nonESAs. Conversely, the maximum achievable tag bandwidth is determined by the ASIC, and can only be reached by using antennas that are not electrically small.

\section{READ RANGe ANALysis AND Discussion}

The read range is defined as the maximum distance at which the RFID reader can detect the backscattered signal from the tag. It can be calculated as [4]

$$
\mathrm{RR}=\frac{\lambda}{4 \pi} \sqrt{\frac{\mathrm{EIRP} \cdot G \cdot \tau}{P_{c}}},
$$

where $\lambda$ is the wavelength and EIRP is the equivalent isotropically radiated power, determined by local country regulations (e.g., $3.3 \mathrm{~W}$ in Europe and $4 \mathrm{~W}$ in USA). $P_{c}$ is the minimum threshold power necessary to activate the RFID chip, $G$ is the gain of the tag antenna, and $\tau$ is the power transmission coefficient, which is related to the power reflection coefficient by $\tau=\left(1-|s|^{2}\right)$. It can be observed in (9) that the read range is proportional to the square root of $\tau$. This indicates that a significant reduction (to some extent) of the conjugate matching level at the tag resonance might not involve an important degradation of the read range. The ratio between the peak read ranges (achieved at the tag resonance [4]) inferred using $\tau=1-\alpha_{0}^{2}$ (halfpower bandwidth upper bound) and $\tau=1$ (conjugate matching case) in (9), namely

$$
\frac{\mathrm{RR}_{\mathrm{ub}}^{\text {peak }}}{\mathrm{RR}_{\text {conj }}^{\text {peak }}}=\frac{2 \sqrt{\gamma}}{\gamma+1},
$$




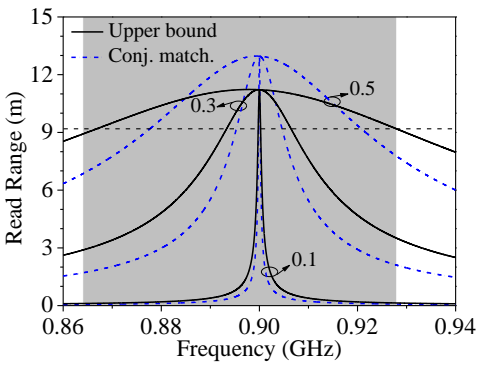

Fig. 3. Simulated read range of small tags exhibiting upper bounds on the half-power bandwidth with (black) and without (blue) conjugate matching for $k_{0} a=0.1,0.3,0.5$. The dashed line indicates the half-power read range level $\left(\mathrm{RR}_{\mathrm{conj}}^{\text {peak }} / \sqrt{2}\right)$. The UHF-RFID band is highlighted in gray.

reveals that the aforementioned $41 \%$ increase of the upper bounds on the half-power bandwidth of a small tag compared to the conjugate matching case (see Section II) involves a reduction of $13.4 \%$ in the peak read range. Expression (10) was obtained assuming EIRP, $P_{c}$, and $G$ to be equal in both cases. This can be appreciated in Fig. 3, where a read range plot of square-shaped (i.e., assuming $\zeta=5.2$ ) small UHF-RFID tags, considering both upper bounds on the halfpower bandwidth with and without conjugate matching, is shown for different antenna sizes $\left(k_{0} a=0.1,0.3\right.$ and 0.5$)$. It was obtained with the help of (9) considering EIRP $=4 \mathrm{~W}, G=\eta D$ (assuming $\eta=1$ and $D=1.5$, i.e., directivity of an elementary electric or magnetic dipole [13]). The power reflection coefficient was obtained from simulation (Keysight $A D S$ ) of the circuit of Fig. 1 using the Alien Higgs 3 chip $\left(G_{c}=1 / 1500 \Omega^{-1}, C_{c}=0.9 \mathrm{pF}\right.$, and $P_{c}=-17 \mathrm{dBm}$, according to the manufacturer). As expected, approximately (notice that RR also depends on $\lambda$ ) a $41 \%$ higher bandwidth evaluated at the half-power read range level (see Fig. 3) and a 13.4\% reduction on the peak read range is obtained, in agreement with the value predicted by (8) and (10), respectively. Notice also that the read range of the upper bound tag becomes flatter within the UHF-RFID frequency band, compared to the conjugate-matched tag.

For a real tag, deviations from the ideal quality factor $\left(Q_{\mathrm{lb}}\right)$ towards higher values are expected. Also, deviations from the ideal equivalent circuit model for the tag antenna (an $R L C$ parallel-circuit) are common. Consequently, the resulting bandwidth is typically far from its upper bound. Bandwidth of a small real tag presenting conjugate matching at resonance can be approximated by (7a) when $Q_{\mathrm{lb}}$ is replaced by the quality factor of the tuned antenna [8]. In virtue of the susceptance slope concept [24], bandwidth of small real tags designed to exhibit proper antenna conductance $\left(G_{c} / \gamma\right)$ for bandwidth optimization can be approximated by (6a), replacing $Q_{\mathrm{lb}}$ by $Q$ of the tuned antenna. Therefore, (8) and (10) are approximately valid for real tags of same size, provided $Q$ and $G$ are equal in both tags under comparison.

UHF-RFID chips exhibit strongly capacitive input impedance and small resistance (the real part being about an order of magnitude smaller than the imaginary part) [3]. This forces the tag antenna to exhibit an inductive reactance much higher than the resistance at $\omega_{0}$, i.e., $X_{a}\left(\omega_{0}\right) \gg R_{a}\left(\omega_{0}\right)$, in order to achieve proper impedance matching. Thus, the equivalent antenna conductance and susceptance of a general tag antenna evaluated at $\omega_{0}$ can be approximated by $G_{a}\left(\omega_{0}\right) \approx R_{a}\left(\omega_{0}\right) / X_{a}^{2}\left(\omega_{0}\right)$ and $B_{a}\left(\omega_{0}\right) \approx-1 / X_{a}\left(\omega_{0}\right)$, respectively. Of course, the reactance of the tuned antenna is equal to zero at the tuned frequency $\left(\omega=\omega_{0}\right)$, and $R_{a}\left(\omega_{0}\right)=R_{c}$ ( $R_{c}$ being the real part of the chip impedance) for conjugate matching. Equivalently, the susceptance $B_{0}$ of the tuned antenna is equal to zero at the tag resonance, and the conductance of the tuned antenna satisfies $G_{0}\left(\omega_{0}\right)=G_{a}\left(\omega_{0}\right)=G_{c}$ for perfect matching. It directly follows that $G_{c} \approx R_{c} / X_{a}^{2}\left(\omega_{0}\right)$. Therefore, by forcing the antenna resistance to be $R_{a}\left(\omega_{0}\right)=R_{c} / \gamma$, one obtains that $G_{a}\left(\omega_{0}\right) \approx G_{c} / \gamma$ and, consequently, bandwidth enhancement with respect to the conjugate matching case, approximately given by (8), is expected. The input resistance of symmetrically fed small wire antennas (commonly used for tag design) is in general much lower than the chip resistance $R_{c}$, giving rise to a significant impedance mismatch. However, it is possible to increase the input resistance to obtain good impedance matching by changing the chip position, without the need of an external matching network [13], [28]. This procedure avoids lowering $\eta$ for bandwidth enhancement (a commonly used technique in practice), allowing the design of efficient small tags with high read range.

\section{PRACTICAL EXAMPLE}

Two small RFID tags (same size) based on a split-ring resonator (SRR) antenna and a commercial integrated ASIC (the Alien Higgs 3 with SOT-323 packaging) were designed (by means of the Keysight Momentum commercial software) for bandwidth comparison purposes. The input impedance of this chip provided by the manufacturer is $Z_{c}=25-j 193 \Omega$ at $f_{0}=900 \mathrm{MHz}$. The following points were considered as design guidelines: (1) bandwidth broadening was forced at the half-power level $(\alpha=1 / 2)$, (2) the tag antenna was forced to be electrically small; meaning that $k_{0} a<0.5$, (3) the available ASIC with the lowest sensitivity was selected, since it provides higher read range without affecting the upper bounds on bandwidth within the ESA region, (4) the efficiency of the antenna was forced to be high enough to ensure operating within the ESA region (i.e., $k_{0} a_{1} \geq k_{0} a$ ).

The Arlon CuClad $250 L X$ with thickness $h_{s}=0.49 \mathrm{~mm}$, relative permittivity $\varepsilon_{r}=2.42$, and loss tangent $\delta_{\text {loss }}=0.0018$, was selected as a substrate. We first design a conjugate-matched tag following the design procedure presented in [28]. The dimensions of the tag antenna (see Fig. 4a) are $a=16.5 \mathrm{~mm} \quad\left(\lambda_{0} / 20, \quad k_{0} a=0.31\right)$, $c=2.2 \mathrm{~mm}, d=0.5 \mathrm{~mm}$, and the slit width $2.2 \mathrm{~mm}$. The chip position was found to be $\varphi_{p}=130^{\circ}$. A second tag was designed for half-power bandwidth enhancement by forcing the antenna resistance to be $R_{a}\left(\omega_{0}\right)=R_{c} / \gamma=25 / 3 \Omega$. To this end, the chip position was changed to $\varphi_{p}=76^{\circ}$, and the distance between rings was adjusted to $d=0.28 \mathrm{~mm}$ in order to maintain resonance at $f_{0}$.

The simulated results for the conjugate-matched tag and the tag with improved bandwidth are summarized in Table II. The efficiency is similar in both cases and much higher than the minimum value ( 0.19 in this case) to ensure operation within the first region. It can be also seen that the quality factor of the improved antenna is slightly higher, due to small differences in the geometry (i.e., distance between rings changes). The simulated power reflection coefficient $|s|^{2}$ of the designed tags is depicted in Fig. 4(b). The improved tag reaches $12.9 \mathrm{MHz}$ half-power bandwidth, i.e., a 37\% bandwidth increase with respect to the conjugate-matched case $(9.4 \mathrm{MHz})$, which is very close to the expected value (41\%), inferred using (8). Such small difference is mainly attributed to the fact that the quality factor of the antenna with improved bandwidth is somewhat higher. The simulated fractional bandwidth is very close to the predicted value for the perfect-matched $(1.07 \%, 9.6 \mathrm{MHz})$ and the improved $(1.42 \%, 12.8 \mathrm{MHz}) \mathrm{tag}$, inferred respectively from (6a) and (7a), replacing $Q_{\mathrm{lb}}$ by $Q_{Z}$. According to (6a) and (7a), the upper bounds on the tag half-power fractional bandwidth are $\mathrm{FBW}_{\mathrm{ub}}=3.4 \%$ $(30.6 \mathrm{MHz})$ and $\mathrm{FBW}_{\text {conj,ub }}=2.4 \%(21.7 \mathrm{MHz})$. It was considered $\eta=0.7$ and $\zeta=9 \pi / 8$ (corresponding to planar antennas with a circular disk shape) which leads to $Q_{\mathrm{lb}}=83$, that is significantly smaller than $Q_{Z}$ for both tags. This suggests that further increase of the tag bandwidth can theoretically be obtained maintaining the same maximum antenna dimension and shape, by simply restructuring the tag antenna to reduce its interior fields and therefore its $Q$ (which is out of the scope of this paper) [8]. Of course, as Fano shows [29] and Sievenpiper illustrates [17], designing an antenna with multiple resonances and avoiding a perfect match can exceed the upper bounds on bandwidth given in (7), within the Bode limit [11]. 


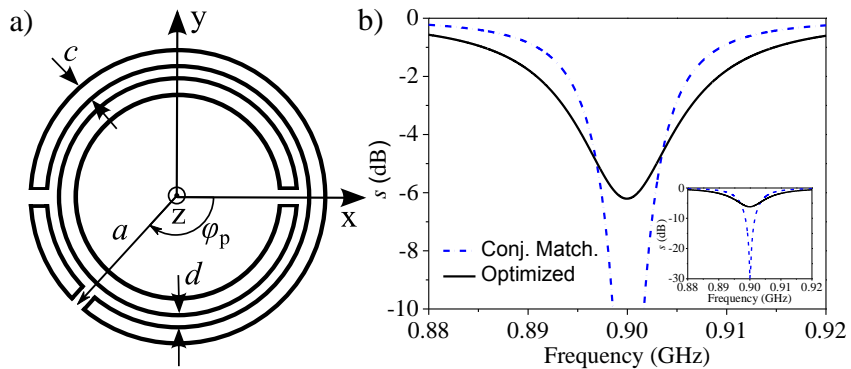

Fig. 4. (a) Topology of the SRR. The cut at the bottom of the external ring indicates the chip position. (b) Simulated power reflection coefficient of the designed tags.

TABLE II

Simulated Results OF THE Designed TAGS

\begin{tabular}{lccccc}
\hline \hline & $\boldsymbol{k}_{\boldsymbol{0}} \boldsymbol{a}$ & $\boldsymbol{\eta}$ & $\boldsymbol{Q}_{\mathrm{z}}$ & HP-FBW & $\mathbf{R R}^{\text {peak }}(\mathbf{m})$ \\
\hline $\begin{array}{l}\text { Conj. } \\
\text { Match. }\end{array}$ & 0.31 & 0.7 & 187.3 & $1.04 \%(9.4 \mathrm{MHz})$ & 11.2 \\
\hline Improved & 0.31 & 0.68 & 198.1 & $1.43 \%(12.9 \mathrm{MHz})$ & 9.8 \\
\hline \hline
\end{tabular}
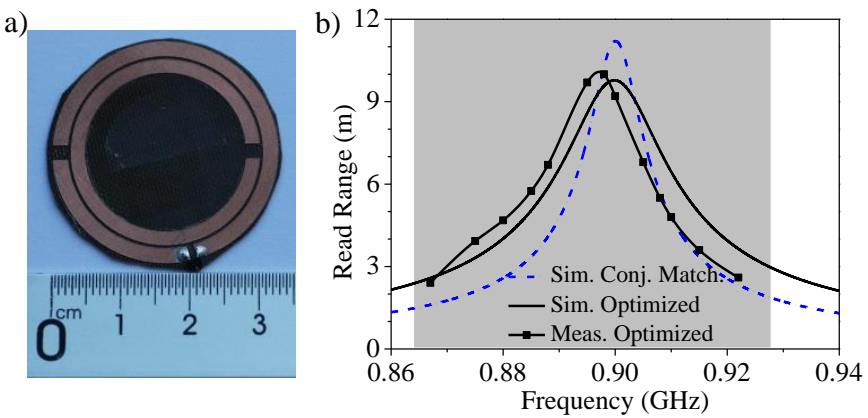

Fig. 5. (a) Photograph of the fabricated tag and (b) simulated and measured read range. A simulation of the conjugate-matched tag is also shown for comparison purposes. The gray region indicates the UHF-RFID band.

The designed tags behave as small current loops providing a dipole-like radiation pattern within the entire UHF-RFID band [28]. The simulated read ranges (see Fig. 5b) were obtained from (9) assuming a constant gain over frequency with $D=1.6$ and $\eta=0.7$ (obtained from electromagnetic simulation at $f_{0}$ ). It can be seen that the read range of the optimized tag was significantly improved in most of the band presenting a peak value of $9.8 \mathrm{~m}$, i.e., a $12.5 \%$ reduction with respect to the perfect-matched tag, which is very close to the expected $13.4 \%$. The tag designed to enhance the half-power bandwidth was fabricated (see Fig. 5a) and the experimental read range was obtained in a TEM cell environment as reported in [28]. Simulated and measured results (Fig. 5b) are in good agreement, showing a small frequency shift of $2 \mathrm{MHz}$ between them. The experimental read range reaches a peak value of approximately $10 \mathrm{~m}$, which is very close to the value obtained from electromagnetic simulation $(9.8 \mathrm{~m})$. Moreover, the experimental read range halfpower bandwidth $(12 \mathrm{MHz})$, is close to the simulated value $(12.9 \mathrm{MHz})$. The difference is mainly attributed to variations on the tag antenna gain over frequency (not considered in the simulated read range) and frequency variations of the chip sensitivity [30].

\section{CONCLUSION}

In this paper, the upper bounds on the bandwidth of single resonant UHF-RFID tags related to the maximum tag dimension, with and without considering conjugate matching, have been explored. We have found that the antenna determines bandwidth upper bounds of small tags $\left(k_{0} a<0.5\right)$, see $(7 a)$, whereas both the ASIC and the antenna determine bandwidth upper bounds for nonESAs. Conversely, the maximum achievable tag bandwidth, given by (7c), is determined by the ASIC, and can only be reached by using antennas that are not electrically small. It has been shown that the upper bounds on the bandwidth of small tags are substantially higher with respect to the case of considering perfect matching at resonance. Specifically, the tag half-power bandwidth is $41 \%$ higher, requiring a relaxation of the conjugate matching condition at the tag resonance in an appropriate manner. Moreover, two SRR-based tags of same size ( $k_{0} a=0.31$ ) has been designed to show that bandwidth of small real tags with conjugate matching (typically much smaller than its upper bound) can also be improved approximately the same factor by simply changing the chip position, at the expense of a small reduction $(13.4 \%)$ of the peak read range. The enhanced tag has also been fabricated and measured, as a proof of concept. The analysis presented in this work provide RFID designers with the capability of determining how well a tag performs in terms of bandwidth, compared to theoretical upper bounds.

\section{APPENDIX}

THE KARUSH-KHUN-TuCKer METHOD

Let us consider the fractional bandwidth function (5) to be maximized for a given RFID ASIC, subject to the following constraints: $Q_{\mathrm{ap}} \geq Q_{\mathrm{b}}, G_{\mathrm{ap}}>0$ and $C_{\mathrm{ap}} \geq 0$. This problem can be simplified by considering a new function $f$, resulting from squaring the product between the right hand side of (5) and $\omega_{0}$, to be maximized, i.e.,

$$
\operatorname{Max} . f\left(G_{\text {ap }}, C_{\text {ap }}\right)=\frac{2 \gamma G_{\text {ap }} G_{c}-G_{\text {ap }}^{2}-G_{c}^{2}}{\left(C_{\text {ap }}+C_{c}\right)^{2}},
$$

subject to the aforementioned necessary conditions or, equivalently,

$$
\begin{aligned}
& g_{1}=\frac{\omega_{0}}{G_{\text {ap }}}\left(C_{\text {ap }}+C_{c}\right)-Q_{\mathrm{bb}} \geq 0 \\
& g_{2}=G_{\text {ap }}>0 \\
& g_{3}=C_{\text {ap }} \geq 0 .
\end{aligned}
$$

To solve this problem by means of the Karush-Kuhn-Tucker (KKT) method [25], [26], we first define the Lagrangian function as

$$
L=f\left(G_{\text {ap }}, C_{\text {ap }}\right)-\sum_{i=1}^{3} \lambda_{i} g_{i},
$$

where $\lambda_{i}$ are the KKT (or Lagrange) multipliers. We then define the KKT conditions as

$$
\begin{aligned}
& \frac{\partial L}{\partial G_{\text {ap }}}=0 \\
& \frac{\partial L}{\partial C_{\text {ap }}}=0 \\
& \lambda_{i} g_{i}=0 .
\end{aligned}
$$

These conditions give rise to the following equations

$$
\begin{aligned}
& \frac{2 \gamma G_{c}-2 G_{\text {ap }}}{\left(C_{c}+C_{\text {ap }}\right)^{2}}+\lambda_{1} \frac{\omega_{0}}{G_{\text {ap }}^{2}}\left(C_{c}+C_{\text {ap }}\right)-\lambda_{2}=0 \\
& \frac{2\left(G_{\text {ap }}^{2}+G_{c}^{2}-2 \gamma G_{\text {ap }} G_{c}\right)}{\left(C_{c}+C_{\text {ap }}\right)^{3}}-\lambda_{1} \frac{\omega_{0}}{G_{\text {ap }}}-\lambda_{3}=0 \\
& \lambda_{1}\left[\frac{\omega_{0}}{G_{\text {ap }}}\left(C_{\text {ap }}+C_{c}\right)-Q_{\mathrm{lb}}\right]=0 \\
& \lambda_{2} G_{\text {ap }}=0 \\
& \lambda_{3} C_{\text {ap }}=0 .
\end{aligned}
$$


TABLE A.I

SOLUTIONS OF THE SYSTEM OF EQUATIONS (A.5)

\begin{tabular}{ccccc}
\hline \hline $\boldsymbol{G}_{\text {ap }}$ & $\boldsymbol{C}_{\text {ap }}$ & $\lambda_{1}$ & $\lambda_{2}$ & $\lambda_{3}$ \\
\hline$\frac{G_{c}}{\gamma_{0}}$ & $\frac{G_{c} Q_{\mathrm{lb}}}{\gamma_{0} \omega_{0}}-C_{c}$ & $\frac{2 \omega_{0}^{2}}{Q_{\mathrm{lb}}^{3}}\left(1-\gamma_{0}^{2}\right)$ & 0 & 0 \\
$\frac{\omega_{0} C_{c}}{Q_{\mathrm{lb}}}$ & 0 & $\frac{2 \omega_{0}^{2}}{Q_{\mathrm{lb}}^{3}}-\frac{2 \gamma_{0} \omega_{0} G_{c}}{C_{c} Q_{\mathrm{lb}}^{2}}$ & 0 & $\frac{2 G_{c}^{2}}{C_{c}^{3}}-\frac{2 \gamma_{0} \omega_{0} G_{c}}{Q_{\mathrm{lb}} C_{c}^{2}}$ \\
$\gamma_{0} G_{c}$ & 0 & 0 & 0 & $\frac{2 G_{c}^{2}}{\left(C_{c}+C_{\mathrm{ap}}\right)^{3}}\left(1-\gamma_{0}^{2}\right)$ \\
\hline \hline
\end{tabular}

Three points were found to be solutions of (A.5) and are summarized in Table A.I. Such points must simultaneously satisfy $\lambda_{i} \leq 0$ and the necessary conditions $Q \geq Q_{\mathrm{lb}}, G_{a}>0$, and $C_{a} \geq 0$, according to the KKT method. An inspection of the results shown in Table A.I reveals that the first point (top row in Table A.I) is a valid solution provided that $C_{\mathrm{ap}} \geq 0$ or, equivalently, $Q_{\mathrm{lb}} \geq \gamma Q_{c}$. The second point (middle row in Table A.I) becomes a valid solution provided that $\lambda_{1} \leq 0$ and $\lambda_{3} \leq 0$ or, alternatively, $Q_{c} \gamma \leq Q_{\mathrm{lb}} \leq \gamma Q_{c}$. Finally, the third point (bottom row in Table A.I) results in a real solution for the optimization problem provided that $\lambda_{3} \leq 0$ or, identically, $Q_{\mathrm{lb}} \leq Q_{c} / \gamma$.

\section{REFERENCES}

[1] K. Finkenzeller, RFID Handbook: Radio-Frequency Identification Fundamentals and Applications, 2nd ed: Wiley, 2004

[2] GS1 EPCglobal, Regulatory status for using RFID in the EPC Gen 2 band ( 860 to $960 \mathrm{MHz}$ ) of the UHF spectrum, 30 Nov. 2016.

[3] G. Marrocco, "The art of UHF-RFID antenna design: impedance matching and size-reduction techniques," IEEE Antennas Propag. Mag., vol. 50, no. 1, pp. 66-79, Feb. 2008.

[4] K. V. Seshagiri Rao, P. V. Nikitin, and S. F. Lam, "Antenna design for UHF-RFID tags: a review and a practical application," IEEE Trans. Antennas Propag., vol. 53, pp. 3870-3876, Dec. 2005.

[5] H. A. Wheeler, "Fundamental limitations of small antennas," in Proc. IRE, 1947, pp. 1479-1484.

[6] L. J. Chu, "Physical limitations of omni-directional antennas," J. Appl. Phys., vol. 19, pp. 1163-1175, Dec. 1948.

[7] R. C. Hansen, "Fundamental limitations in antennas,"in Proc. IEEE, 1981, pp. 170-182.

[8] A. D. Yaghjian and S. R. Best, "Impedance, bandwidth, and Q of antennas," IEEE Trans. Antennas Propag., vol. 53, no. 4, pp. 12981324, April 2005.

[9] M. Gustafsson, C. Sohl, and G. Kristensson, "Physical limitations on antennas of arbitrary shape," in Proc. Roy. Soc. A, vol. 463, pp. 25892607, 2007.

[10] G. Zamora, F. Paredes, F. J. Herraiz-Martinez, and F. Martin and J. Bonache "Bandwidth limitations of UHF-RFID tags," IET Microw. Antennas Propag., vol. 7, no. 10, pp. 788-794, July 2013.

[11] H. W. Bode, "Network analysis and feedback amplifier design," pp. 360-371, D. Van Nostrand co., N.Y., 1945.

[12] E. Bergeret, J. Gaubert, P. Pannier and J.M. Gaultier, "Modeling and design of CMOS UHF voltage multiplier for RFID in a EEPROM compatible process," IEEE Trans. Circuits Syst. II, Exp. Briefs, vol. 54, no. 10, pp.833-837, Oct. 2007.

[13] C.A. Balanis, Antenna theory: analysis and design, 3rd ed., John Wiley \& Sons Inc., New Jersey, 2005.

[14] R. A. Foster, "A reactance theorem," Bell Syst. Tech. J., vol. 3, pp. 259 267, 1924.

[15] G. Sisó, M. Gil, J. Bonache and F. Martin, "On the dispersion characteristics of metamaterial transmission lines," J. Appl. Phys., vol. 102, no. 7, pp. 074911 (1-7), Oct. 2007.

[16] J. S. McLean, "A re-examination of the fundamental limits on the radiation Q of electrically small antennas," IEEE Trans. Antennas Propag., vol. 44, no. 5, pp. 672-676, May 1996.

[17] D. F. Sievenpiper, D. C. Dawson, M. M. Jacob, T. Kanar, S. Kim, J. Long, and R. G. Quarfoth, "Experimental Validation of Performance Limits and Design Guidelines for Small Antennas," IEEE Trans. Antennas Propag., vol. 60, no. 1, pp. 8-19, Jan. 2012.
[18] G. A. E. Vandenbosch, "Explicit relation between volume and lower bound for Q for small dipole topologies," IEEE Trans. Antennas Propag., vol. 60, no. 2, pp. 1147-1152, Feb. 2012.

[19] M. Gustafsson, M. Cismasu, and B. L. G. Jonsson, "Physical bounds and optimal currents on antennas," IEEE Trans. Antennas Propag., vol. 60, no. 6, pp. 2672-2681, June 2012.

[20] M. Gustafsson, C. Sohl, and G. Kristensson, "Illustrations of New Physical Bounds on Linearly Polarized Antennas," IEEE Trans. Antennas Propag., vol. 57, no. 5, pp. 1319-1327, May 2009.

[21] A. D. Yaghjian and H. R. Stuart, "Lower Bounds on the Q of Electrically Small Dipole Antennas," IEEE Trans. Antennas Propag., vol. 58, no. 10, pp. 3114-3121, Oct. 2010.

[22] K. Mohammadpour-Aghdam, R. Faraji-Dana, G. A. Vandenbosch, S. Radiom, and G. G. Gielen, "Physical bound on Q factor for planar antennas," in Proc. 41 $1^{\text {th }}$ Eur. Microw. Conf., Oct. 2011.

[23] K. Kurokawa, "Power waves and the scattering matrix," IEEE Trans. Microw. Theory Tech., vol. 13, no. 2, pp. 194-202, March 1965.

[24] J. S. G. Hong and M. J. Lancaster, Microstrip Filters For RF/Microwave Applications, Wiley, 2004.

[25] W. Karush, "Minima of Functions of Several Variables with Inequalities as Side Constraints," M.S. thesis, Dept. Math., Chicago Univ., Chicago, Illinois, 1939

[26] H. W. Kuhn and A. W. Tucker, "Nonlinear Programming," in Proc. 2nd Berkeley Symp., pp. 481-492, 1951.

[27] J. Xi and H. Zhu, "UHF RFID Impedance Matching T-Match-Dipole Tag Design on the Highway," IEEE Int. Conf. RFID, pp. 86-93, 2015.

[28] S. Zuffanelli, G. Zamora, P. Aguilà, F. Paredes, F. Martín, and J. Bonache, "Analysis of the Split Ring Resonator (SRR) Antenna Applied to Passive UHF-RFID Tag Design", IEEE Trans. Antennas Propag., vol. 64, no. 3, pp. 856-864, March 2016.

[29] R. M. Fano, Theoretical limitations on the broadband matching of arbitrary impedances, Res. Lab. Electron., MIT Technol. Rep. 41, Jan. 2, 1948.

[30] P. V. Nikitin, K. V. Seshagiri Rao, R. Martinez, S. F. Lam, "Sensitivity and impedance measurements of UHF-RFID chips," IEEE Trans. Microw. Theory Tech., vol. 57,no. 5, pp. 1297-1302, May 2009. 